\title{
Search Cost and Obfuscation Equilibrium: An Empirical Investigation
}

\author{
Yiyu Chen ${ }^{1, *}$ \\ ${ }^{1}$ Business School, Nankai University, Tianjin 300071, China \\ *Corresponding author. Email: yiyuchen820@gmail.com
}

\begin{abstract}
Easy price search makes demand tremendously price-sensitive. However, merchants' obfuscation strategy can hinder consumers from searching, thus greatly reducing their price sensitivity to other products. This paper presents an empirical examination of how obfuscation strategy affects consumers' purchasing decisions. The author improves the model proposed by Glenn Ellison and Sara Fisher Ellison and applies the observed data from taobao.com and tmall.com to the model. This paper proves that, for some products in these two websites, the prices and the rankings can significantly affect sales volume, and for some products, merchants will adopt obfuscation strategies for low-quality products to maximize their profits.
\end{abstract}

Keywords: Obfuscation, Search cost, Advertising, Customer welfare.

\section{INTRODUCTION}

With the development of the Internet and digital economy, Internet technology will make it easy for consumers to find the exact product they want and compare prices from rival retailers. The most intriguing demonstration of this potential so far is the price search engine. However, it is common for merchants to control search costs and prices in order to boost profits. Anything that increases understanding of the cost of each firm's products or forces more consumers to search firm-by-firm is likely to be seen as a form of obfuscation.

Obfuscation often occurs in practice. Instead of creating artificial search friction, for instance, supermarkets use limited shelf space to place products with different profit margins. One phrase commonly used is "eye level is buying level". In Mitford(1978)'s book[1], she describes how the coffin sellers first guide customers to buy more expensive items, and then introduce a cheaper alternative to consumers.

This paper aims to explore how obfuscation strategy affects consumers' purchasing decisions. For this purpose, the author takes a simple model originally presented in Stahl(1989)[2], and was further refined by G. Ellison and S.F. Ellison(2009)[3]. Ellison and Ellison studied the competition between a group of Internet retailers operating in an environment dominated by price search engines. The author made an empirical analysis using relevant data on monitor, mouse and keyboard, which were obtained from Taobao and Tmall under Alibaba Group.

Predecessors to $\mathrm{C} 2 \mathrm{C}$ websites such as Taobao and Tmall research mainly includes three aspects, one is from reputation, information transfer and market contract execution, to explore the effectiveness of the e-commerce market, the second is to explore the management mode of e-commerce trade platform itself and competitive strategy, the third is the study of the characteristics of online consumer behavior and analyze the causes. For instance, Weian $\mathrm{Li}$, Desheng $\mathrm{Wu}$ and Hao $\mathrm{Xu}$ (2007)[4] and Youzhi Xue and Yongfeng Guo(2012)[5] from the seller's perspective, using the data of Taobao, studies the competitive strategy. Yali Dong and Bei Yang(2007)[6], Puyang Sun, Jingjia Zhang and Xiaoyu Jiang(2017)[7] studied the factors that influence consumers' purchasing behavior.

On the basis of existing research, this paper contributes to the recent economic literature on obfuscation by examining the topic in two websites as a mechanism for screening consumer preferences. 
Through empirical results, it can be proved that both rank and price will have a negative impact on sales. But in some obfuscation strategies, the price can also be positively correlated with volume. Product rankings have a significant impact on sales. Obfuscation strategies will bring greater profits to merchants. At the same time, this paper also proves that the price of low- and medium-quality products on Taobao has a significant impact on the sales of products, while Tmall, on the contrary, only highquality products have this situation. Second, in different models of the same product, merchants will adopt different obfuscation strategies for lowquality products to maximize profits.

The rest of this paper is organized as follows. The using model and theory are listed in Section 2. Section 3 puts forward two hypotheses. In Section 4, the author discusses the collected data, studies the impact of obfuscation and price on the sales volume of products of different qualities, verifies the hypothesis. Section 5 concludes.

\section{THE MODEL}

This section begins with a simple model presented by Stahl in 1989. The author then discusses a more specific micro model presented by G. Ellison and S.F. Ellison(2009) under the incomplete consumer search, allowing search friction to be determined by the competing investments of search engines and retailers.

\subsection{The Forerunner Model}

Stahl(1989), considers a model with two types of consumers. The consumer with the proportion $\mu \in[0,1]$ has zero search cost; these consumers were referred to "shoppers". The remaining $1-\mu$ proportion of consumers have a common search $\operatorname{cost}^{c}>0$. The Bertrand(1883)[8] result and the Diamond(1971)[9] result obtain in the model when, $\mu=1$ and $\mu=0$, respectively. When $\mu \in[0,1]$, there is no pure-strategy Nash Equilibrium(NE), but there is a unique symmetric NE in mixed strategies. As a result, Stahl's model generates price dispersion. Moreover, with the change of ${ }^{\mu}$ from 0 to 1 , the degenerate distribution $F(p)$ of NE continuously changes from monopoly price (Diamond) to zero price (Bertrand). Further, as the search cost ${ }^{C}$ approaches zero (given $\mu<1$ ), NE converges to the degenerate distribution when the price is zero. These two features achieve the goal of narrowing the difference between Bertrand and Diamond results.

Stahl notes that this model has a unique symmetric equilibrium: retailers randomly allocate prices within a certain range; informed consumers purchase from the firm that offers the lowest price; others will buy from the first website they visit, if the website's price falls below a certain threshold (which is always in equilibrium). The solution is continuous in ${ }^{\mu}$ and varies in the manner assumed above if define $s \equiv 1-\mu$ : retailer profits are lower and demand is higher (in the sense of firstorder random advantage) when the fraction of informed consumers is higher, and price converges to cost in the limit as ${ }^{\mu}$ goes to one. The solution has similar comparative statics in the ${ }^{c}$ parameter, so one could alternately take $s \equiv c$ as the measure of search frictions.

\subsection{Incomplete Consumer Search}

G. Ellison and S.F. Ellison(2009) constructs a more specific micro model, allowing search friction to be determined by the competing investments of search engines and retailers.

It is a clear model of friction for research purposes, assuming that the ability of consumers to absorb the information displayed on the webpage is different. According to Ellison and Ellison, the search engine's investment $x^{s e}$ would be an investment in collecting information and presenting it in a manner that is easier to understand. Retailers' investments $x^{r}$ would be investments in making pricing more complicated and difficult to describe. To fit the model above exactly, one could assume that the distribution of consumer abilities is such that after these investments are made, a fraction $\mu_{0}+x^{s e}-x^{r}$ of consumers are able to understand the webpage fully and thereby learn all firms' prices, whereas the rest of the consumers cannot understand the price information at all and must resort to visiting the listed websites sequentially (in random order) to understand each retailer's price. The analysis of the search and obfuscation game would then follow exactly as described above. In any interior equilibrium, the model shows a positive increase in retail sales, price dispersion across retailers, and limited knowledge of prices among some consumers. 
Based on G. Ellison and S.F. Ellison's model, to provide a straightforward analysis of how consumers choose low-, medium- and high-quality between site $\mathrm{A}$ and site $\mathrm{B}$, the author estimates simple logit models on the consumer-level data. $Q_{\text {wqt }}=e^{X_{\text {wqt }} \beta}$

with
Formally, the author supposes that customer choosing to buy a quality $q$ product, the quantity $Q$ of products purchased from the website ${ }^{W}$ on the day ${ }^{t}$ is

$X_{\text {wqt }} \beta=\beta_{0}+\beta_{1} \log \left(1+\operatorname{Rank}_{w q t}\right)+\beta_{2} \log \left(P_{\text {wqt }}\right)+\sum_{t=1}^{30} \beta_{2+t}$ TimeTrend $_{t}+\epsilon_{w}$

In this equation, the dependent variable is the sales volume. In the past days $t \in[1,30]$, customers search and purchase the product of quality $q$ from different websites $w . \epsilon_{w}$ is a standard logit error. Rank wqt reflects the website's position on the data list for the category. Specifically, it represents the average 30-day ranking in which each item is displayed in the search interface when a consumer searches for a monitor, mouse, and keyboard on a website. Display order is an important embodiment of obfuscation strategy. ${ }{ }_{\text {wqt }}$ is the average 30-day price of different categories of products on different

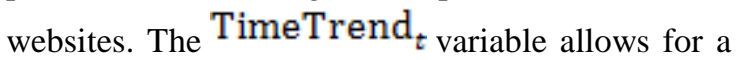
piecewise linear time trend with a slope that changes every 30 days. The author treats $\log \left(1+\operatorname{Rank}_{\text {wqt }}\right)$ as a continuous variable and computes estimated elasticities when all variables are at their means by setting the derivative of Rank $_{\text {wqt }}$ with respect to a change in ${ }_{\text {wqt }}$ equal to the inverse of the average distance between prices. Note that if consumers are fully informed, one would expect that $\beta_{1}$ would be zero and that $\beta_{2}$ would be negative.

\section{THE HYPOTHESIS}

Based on the model, the author believes that in the e-commerce market, for different websites and different products, obfuscation will have different manifestations.

On this basis, the author proposes two hypotheses. The first is about the performance of different websites. As a strategy to increase the search cost of consumers, obfuscation should be based on a certain degree of user stickiness. Newer sites may not adopt such strategies too often to avoid losing customers.
H1 For a more mature site, obfuscation occurs and affects more.

The second hypothesis is about products. In marketing, businesses often change the price, advertisement or quality of some products to achieve the purpose of selling the other products. Therefore, the author supposes that in the same product, different quality and different price models will be adopted different obfuscation strategies.

$\mathrm{H} 2$ For the same product category, different quality will also be obfuscated by merchants, and it is most significant in low-quality products.

In order to verify the above two hypotheses, the author made regression analyses in section 4 .

\section{EMPIRICAL ANALYSIS}

\subsection{Data}

The data used by this paper were downloaded from TAOBAO.com and TMALL.com. The author considers that Alibaba's Taobao and Tmall are very typical examples of obfuscation. According to the 2019 eMarketer report, in 2018, Taobao and Tmall occupied the first and second place of e-commerce platform worldwide ranking, and their gross merchandise value(GMV) reached $\$ 515$ and $\$ 432$ billion respectively. The shopping festivals such as "November 11", "March 8" created by them are typical examples of obfuscation promotion strategies. These data were collected at daily frequency from February 1, 2021 to March 3, 2021. They contain information within each of the eight predefined categories mentioned above.

In this section, the author collected data on three areas: the Alienware monitor, the Logitech mouse, and the HP keyboard. The author considers monitor to be of high quality, mouse to be of medium quality, and keyboard to be of low quality.

The raw data is at the level of sales and indicates from which website the customer bought 
the product, the number of sales, the price and cost, search interface. and the daily average ranking of products in the

Table 1. Summary statistics for monitor, mouse and keyboard data

\begin{tabular}{lccccc}
\hline \multicolumn{1}{c}{ Variables } & $\mathrm{N}$ & Mean & Stdev & Min & Max \\
\hline Qlow & 154 & 10.96 & 61.70 & 0.033 & 666.7 \\
QMid & 125 & 6.536 & 19.34 & 0 & 138.9 \\
QHi & 93 & 0.564 & 1.260 & 0 & 7 \\
Plow & 154 & 131.5 & 25.67 & 89 & 199 \\
PMid & 125 & 662.8 & 243.5 & 369 & 1,349 \\
PHi & 93 & 8,578 & 7,127 & 3,375 & 34,999 \\
$\log \left(1+\operatorname{Rank}_{L}\right)$ & 154 & 2.166 & 0.465 & 0.301 & 2.648 \\
$\log \left(1+\operatorname{Rank}_{M}\right)$ & 125 & 1.945 & 0.605 & 0.301 & 2.600 \\
$\log \left(1+\operatorname{Rank}_{H}\right)$ & 93 & 1.761 & 0.446 & 0.301 & 2.356 \\
\hline
\end{tabular}

The author made descriptive statistics on the data ("Table 1"). Q represents the dependent variable $Q_{w q t}$ in the model. 30 days average sales of low-quality product (keyboard) is Qlow, of medium-quality (mouse) is $Q M i d$, of high-quality (monitor) is $Q H i$. The 30-day average prices are expressed by $P$ low $P M i d$ and $P H i$ respectively. $\log (1+$ Rank $)$ are calculated based on the natural number ranking of the product in the search interface.

It can be seen that the standard deviation of $P H i$ is high. The maximum is 34,999 and the minimum is 3375 . Considering the price of a highquality product, the author thinks the standard deviation is reasonable. About other variables, low-

Table 2. Evidence of incomplete consumer search: effects of product prices and rankings on different products

\begin{tabular}{llll}
\hline \multirow{2}{*}{ Independent Variables } & \multicolumn{2}{c}{ Dep. var.: Sales of each quality level } \\
\cline { 2 - 4 } & Low_q & Mid_q & High_q \\
\hline$\beta_{0}$ & $8.507^{* * *}$ & $4.021^{* * *}$ & 0.417 \\
& $(5.29)$ & $(2.94)$ & $(0.39)$ \\
$\log (1+$ Rank $)$ & $-1.508^{* * *}$ & $-0.702^{* * *}$ & $-0.581^{* * *}$ \\
& $(-11.26)$ & $(-5.53)$ & $(-3.46)$ \\
$\log (P)$ & $-2.650^{* * *}$ & $-1.015^{* *}$ & -0.086 \\
Observations & $(-3.62)$ & $(-2.10)$ & $(-0.30)$ \\
R-squared & 154 & 125 & 93 \\
\hline
\end{tabular}

a The numbers in parentheses are t statistics; * $p<0.1, * * p<0.05, * * * p<0.01$ quality products' price minimum is 89 and the maximum is 199 . The price of the medium-quality product is from 369 to 1349 . The daily sales of the low-quality product are from 0.033 to 666.7 , medium-quality product is from 0 to 138.9 and high-quality product is from 0 to 7 .

\subsection{Results}

The author discusses how the rankings and prices of three different quality products on one website affect sales volume. Comparisons between websites are not considered, so the subscript ${ }^{W}$ on equation (1) is omitted in this table. The investigation results are shown in "Table 2". 
In "Table 2", it can be seen that $\log (1+\operatorname{Rank})$ and $\log (P)$ both have negative effect on the sales. This shows that whether it is a high-, mid- or low-quality products, the display order on the search interface has an important impact on the purchase of consumers. It is reasonable because lower ranking means less search cost, and the more likely consumers will make a purchase if it meets their psychological expectations. The inverse ratio of price and volume is also understandable. This is also the reason why merchants are willing to use obfuscation strategy.

It can be noted that price is significant for sales of low- and medium-quality products at $1 \%$ and Table 3. Evidence of incomplete consumer learning: effects of product prices and rankings on different websites (Taobao and Tmall)

\begin{tabular}{|c|c|c|c|c|c|c|}
\hline \multirow{3}{*}{$\begin{array}{l}\text { Independent } \\
\text { Variables }\end{array}$} & \multicolumn{6}{|c|}{ Dep. var.: Sales of each quality level } \\
\hline & \multicolumn{3}{|c|}{ Taobao.com } & \multicolumn{3}{|c|}{ Tmall.com } \\
\hline & Low_q & Mid_q & High_q & Low_q & Mid_q & High_q \\
\hline \multirow{2}{*}{$\beta_{0}$} & $5.482^{* * *}$ & $5.583^{* * *}$ & $1.676^{\star *}$ & $6.139^{* *}$ & 2.401 & $4.894^{* * *}$ \\
\hline & $(5.21)$ & $(3.24)$ & $(2.35)$ & $(2.48)$ & $(1.46)$ & $(4.20)$ \\
\hline \multirow{2}{*}{$\log (1+\operatorname{Ran}$} & )$^{-1.136^{\star \star \star}}$ & $-1.516^{\star \star \star}$ & $-1.251^{\star * *}$ & $-1.057^{\star * *}$ & $-1.106^{* * *}$ & $-0.569^{\star * *}$ \\
\hline & $(-8.20)$ & $(-6.52)$ & $(-8.35)$ & $(-7.03)$ & $(-3.45)$ & $(-5.81)$ \\
\hline \multirow{2}{*}{$\log (P)$} & $-1.786^{\star \star \star}$ & $-1.333^{\star \star}$ & -0.155 & -1.673 & -0.142 & $-0.985^{\star \star *}$ \\
\hline & $(-4.06)$ & $(-2.32)$ & $(-0.74)$ & $(-1.45)$ & $(-0.24)$ & $(-3.21)$ \\
\hline Observations & 94 & 73 & 70 & 60 & 42 & 41 \\
\hline R-squared & 0.442 & 0.382 & 0.602 & 0.472 & 0.236 & 0.737 \\
\hline
\end{tabular}

The author thinks this is a good result. The $\mathrm{H} 1$ testing results are shown in "Table 3".

In "Table 3", the author compares different websites, Taobao.com and Tmall.com.

In Taobao.com, it can be found that $\log (1+\operatorname{Rank})$ and $\log (P)$ both have negative effect on low-quality product and the coefficient is significant. Rankings also significantly affected sales of high-quality products, but prices remained insignificant.

The results of taobao.com are similar to those presented in "Table 2".

For tmall.com, the ranking is still significant, but the difference is that $\log (P)$ has no significant effect on low quality and medium quality products, but has an effect on high quality products with pvalue less than 0.01 .
$5 \%$, respectively, but not significant for highquality. The author suggests this may be due to differences in price sensitivity. Low-quality products are highly price-sensitive, resulting in a significant impact on sales volume. The price sensitivity of medium-quality products decreases relatively, so its significance decreases. And the price sensitivity of high-quality products is low, so the impact of price is small.

From the model of obfuscation, which is discussed in "Table 2", the rank of products can influence the sales significantly. Moreover, the obfuscation effect can indicate in low-quality product more significant.

The numbers in parentheses are t statistics; * $\mathrm{p}<0.1, * * \mathrm{p}<0.05, * * * \mathrm{p}<0.01$

This confirms H1, more mature Taobao is more consistent with the logic that the phenomenon of low-quality obfuscation is more significant. For the relatively new website Tmall, consumers have relatively low trust in the purchase of high-quality products, so the price sensitivity will be enhanced accordingly. For low to medium quality products, there is also a degree of disorder as a result of trust.

The author continues using equation (1) to test H2. The results are shown in "Table 4". 
Table 4. Evidence of incomplete consumer search: effects of product prices and rankings on different category mouse

\begin{tabular}{llll}
\hline \multirow{2}{*}{ Independent Variables } & \multicolumn{3}{c}{ Dep. var.: mouse's sales of each quality level } \\
& Low_q & Mid_q & High_q \\
\hline$\beta_{0}$ & -3.222 & $2.968^{*}$ & 1.352 \\
& $(-1.30)$ & $(1.90)$ & $(0.53)$ \\
$\log (1+\operatorname{Rank})$ & $-0.768^{* * *}$ & $-0.980^{* * *}$ & $-0.602^{* * *}$ \\
& $(-4.41)$ & $(-6.01)$ & $(-3.78)$ \\
$\log (P)$ & $2.289^{*}$ & -0.492 & -0.171 \\
& $(1.95)$ & $(-0.83)$ & $(-0.20)$ \\
Observations & 122 & 117 & 79 \\
R-squared & 0.188 & $0.241 \quad 0.159$ \\
\hline
\end{tabular}

In "Table 4", the author used three different models of Logitech mouse to represent highquality, medium-quality, and low-quality products: GPW as high-quality product, G502 as mediumquality product and G102 as low-quality product. Rankings still have a significant impact on sales, but the absolute values of the coefficient are reduced compared with the previous analysis.

It is worth noting that in the low-quality case, the price has a positive effect on sales, with a coefficient of 2.289 and a p-value less than 0.1 . Such a positive effect suggests another obfuscation strategy for Logitech's products, making their lowquality products more popular at higher prices. It was a successful obfuscation strategy that magnified their own profits. In another column, the author cannot see some signs in other variables. About the reason, maybe the observation number is limited, if the author wants to see more details, the sample needs to be enlarged.

This will, to some extent, prove the validity of hypothesis 2 . In the same product, merchants did use different obfuscation strategies for different quality models, and the strategy did work for lowquality products.

From the results of table1 to table4, the author can find that $\log (1+\operatorname{Rank})$ and $\log (P)$ are playing important role in obfuscation strategy influence product sales. In Tmall.com, $\log (P)$ also have influence on high-quality product, but not in Taobao. As to different quality level mouse, the author can also verify that $\log (1+$ Rank $)$ have effect on every quality product, and $\log (P)_{\text {has }}$ an anomalously positive effect on sales volume in low-quality models.

\subsection{Robustness Test}

Given the assumed functional form of demand, economic theory implies that prices should be exogenous. In this environment, the profitmaximizing price of the firm with constant marginal cost is independent of the realization of demand shock. Even if a firm observes a demand shock before setting a price, the price set to maximize profits will not be endogenous. But in today's e-commerce landscape, where merchants have access to more information and pricing strategies are more complex, the authors argue that this theory may not hold true. To enhance the rigor, in this section, the author examines the robustness of the two instrumental variable strategies. The price adjustment mechanism of merchants mainly includes: cost (mainly variable cost), supply and demand relationship, price changes of competitors with the same strategic positioning and market segments, and platform policies. In the past, cost was the most important determinant of pricing, but today, cost pricing is less common, and most of the price changes by e-retailers are due to the use of demand information. At the same time, since platform policies are not easy to quantify, the author chose two sets of instruments, which are called the "pageviews" instruments and the "competitor" instruments, to measure the robustness of the model.

The "pageviews" instrument is the contemporaneous total values of pageviews for the products studied (154 HP keyboard, 125 Logitech 
mouse, 93 Alienware monitor). The author uses the $\log (1+\operatorname{Rank}), \log (P)$, and $\log (Q)$ data used in section $4 . \log (V)$ is the logarithm of the pageviews data. The authors used a two-stage least square method to test the endogeneity of this instrument variable. "Table 5" shows the test results of 2SLS. The column "first" represents the first stage and the column "second" represents the second stage.

Table 5. Test results of instrument variables of two-stage least square method

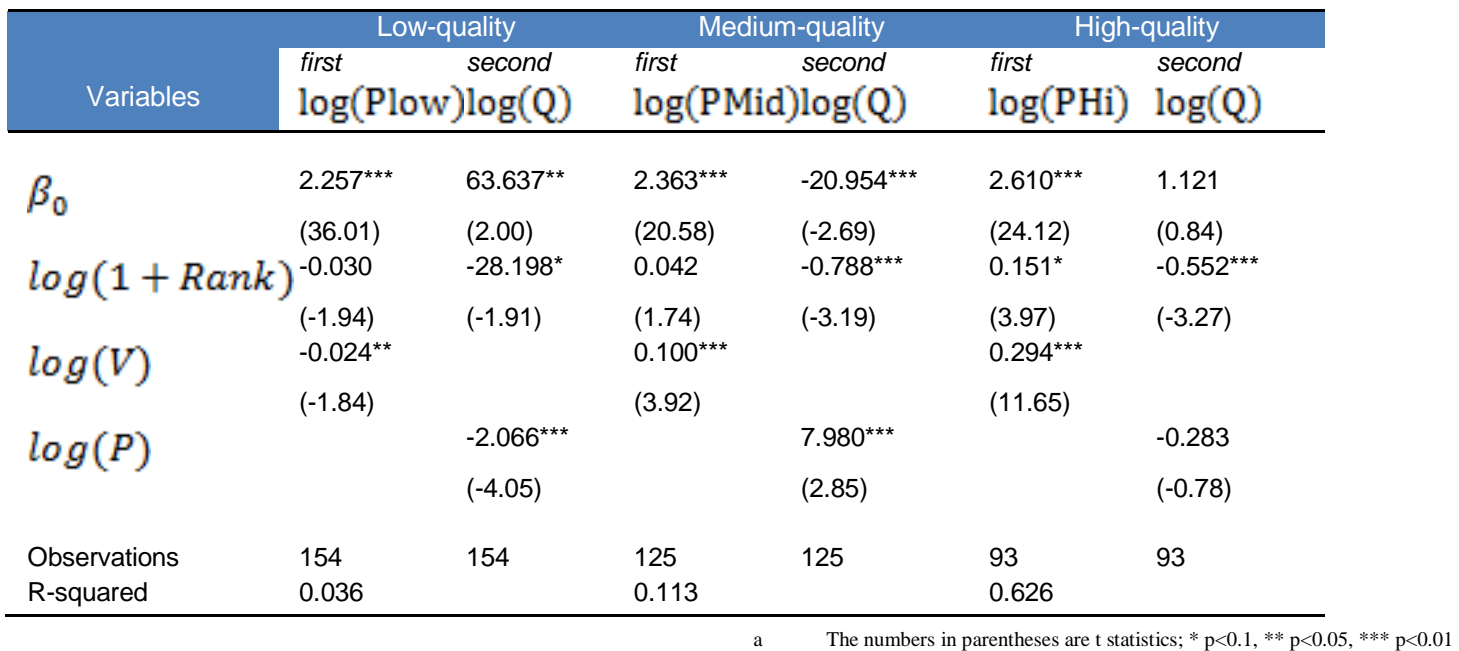

The results show that pageviews do have a significant effect on price. The difference is that in low-quality products, pageviews have a negative impact on price, while in medium- and high-quality products, pageviews have a positive impact. This proves that when merchants formulate sales strategies for low-quality products, they will adopt a low-price strategy for products with greater consumer demand. For medium- and high-quality products, the higher the consumer demand, the higher the price will be set. This is in line with the principle of profit maximization.

For "competitor" instruments, they are the contemporaneous values of $\log (1+$ Rank $)$, $\log (P)$ and $\log (Q)$ for the same website but for different products, Logitech keyboard, Laser mouse and Dell monitor respectively. These three brands have similar strategic positioning to HP, Logitech and Alienware in section 4, with similar price ranges. The authors observed 176 Logitech keyboard, 241 Laser mouse, 118 Dell monitor, and their descriptive statistics are shown in "Table 6". $Q$ is the average daily sales over the 30 -day period, $P$ is the average price over the 30 -day period, and Rank is the ranking of the product in the search interface.

Table 6. Summary statistics for instrumental monitor, mouse and keyboard data

\begin{tabular}{llllll}
\hline \multicolumn{1}{c}{ Variables } & \multicolumn{1}{c}{ N } & \multicolumn{1}{c}{ Mean } & \multicolumn{1}{c}{ Stdev } & \multicolumn{1}{c}{ Min } \\
\hline Qlow & 176 & 10.65 & 38.18 & 0.400 & 333.3 \\
QMid & 241 & 3.070 & 16.04 & 0.0333 & 233.3 \\
QHi & 118 & 1.119 & 2.313 & 0 & 14 \\
Plow & 176 & 195.8 & 57.99 & 111 & 299 \\
PMid & 241 & 231.1 & 72.17 & 109 & 399 \\
PHi & 118 & 4,190 & 1,847 & 2,000 & 10,499 \\
$\log \left(1+\operatorname{Rank}_{L}\right)$ & 176 & 1.833 & 0.390 & 0.301 & 2.248 \\
$\log \left(1+\operatorname{Rank}_{M}\right)$ & 241 & 2.343 & 0.441 & 0.477 & 2.876 \\
$\log \left(1+\operatorname{Rank}_{H}\right)$ & 118 & 1.797 & 0.443 & 0.477 & 2.408 \\
\hline
\end{tabular}


"Table 7" illustrates the effects of product prices and rankings on different instrumental products. It can be clearly seen from "Table 7" that for these competitors, price and ranking also have a significant impact on sales volume, and both price and ranking have a negative impact on sales volume. This is consistent with the research results of this paper. Compared with the result of section 4 ,

Table 7. Effects of product prices and rankings on different instrumental products

\begin{tabular}{|c|c|c|c|}
\hline \multirow{2}{*}{ Independent Variables } & \multicolumn{3}{|c|}{ Dep. var.: Sales of each quality level } \\
\hline & Low_q & Mid_q & High_q \\
\hline \multirow{2}{*}{$\beta_{0}$} & $4.596^{\star \star \star}$ & $4.940^{\star \star \star}$ & $5.269^{\star \star \star}$ \\
\hline & $(7.59)$ & $(7.13)$ & $(6.05)$ \\
\hline \multirow{2}{*}{$\log (1+\operatorname{Rank})$} & $-1.161^{\star \star *}$ & $-0.867^{\star \star *}$ & $-1.142^{\star \star \star}$ \\
\hline & $(-14.35)$ & $(-9.39)$ & $(-11.91)$ \\
\hline \multirow{2}{*}{$\log (P)$} & $-0.929^{\star * *}$ & $-1.429^{\star \star \star}$ & $-1.057^{\star * *}$ \\
\hline & $(-3.72)$ & $(-4.83)$ & $(-4.27)$ \\
\hline Observations & 176 & 241 & 117 \\
\hline R-squared & 0.547 & 0.352 & 0.623 \\
\hline
\end{tabular}

a The numbers in parentheses are t statistics; * $\mathrm{p}<0.1, * * \mathrm{p}<0.05, * * * \mathrm{p}<0.01$

The author believes that this reflects that the instruments eliminate most of the true exogenous variation in these variables. These exogenous changes come from the fact that these prices consider consumer demand and competitor pricing during the adjustment period. The effect of these instruments on prices is stable.

\section{CONCLUSION}

Through empirical results, it can be proved that the ranking of products can significantly affect sales volume. This is consistent with the original hypothesis that obfuscation leads to greater profits for merchants. In addition, in the two hypotheses, for the first one that compares Taobao and Tmall websites, the price of low- and medium--quality products in Taobao also has a significant impact on the sales of the products, but Tmall acts the opposite, which embodies this situation in highquality products. The author speculated that this may be because Taobao's operation time and operation system are more mature, and there are more fixed customer groups. At the same time, there are more medium- and low-quality goods on Taobao. Therefore, when people want to buy medium- and low-quality products, Taobao is the preferred choice. For hypothesis 2, the comparison for high-quality products, price has a more significant impact on sales volume, with p-value less than 0.01. Compared with the result of Section 4, for high-quality products, price has a more significant impact on sales volume, with $\mathrm{p}$ value less than 0.01 . The impact of medium quality prices has also increased in significance. of mouse, the effect of ranking on sales can also be found.

In theory, based on the literature of Stahl and Ellison, this paper proposes another form of an extended model of obfuscation. In existing studies, Weian Li et al.(2007) used the data of Taobao.com to study the role of reputation mechanism in online transactions, Youzhi Xue and Yongfeng Guo(2012) studied Taobao's competitive strategy, Yali Dong and Bei Yang(2007) analyzed the factors that influence consumer buying behavior on Taobao, but they were not from the perspective of obfuscation strategy affects sales, so this paper enriches the empirical conclusion and contribute to the research on obfuscation.

In practice, this paper gives a positive explanation for the obfuscation strategy of online shopping websites with multiple products and merchants, and merchants can learn from the experience of Taobao and Tmall to achieve the goal of profit maximization.

However, this paper also has many shortcomings. First of all, due to the limitations of technical level and platform policy, the data time span and sample size used in this paper are defective, so the empirical results obtained may not be universal. The data may be more convincing at shopping festivals, such as 11 November, when 
obfuscation is most evident. Secondly, this paper only considers the case where merchants obfuscate prices with the products price and the ranking. In fact, there are far more ways to obfuscate prices, for example, personalized price, pre-sell strategy, "deposit first, balance payment later" strategy, cross-store order to get reduction, etc. As merchant obfuscation strategies become more and more abundant, theoretical research should also keep up with the pace, which is also an interesting direction for future research.

\section{AUTHORS' CONTRIBUTIONS} Chen.

This paper is independently completed by Yiyu

\section{ACKNOWLEDGMENTS}

First and foremost, I would like to show my deepest gratitude to the professors, who have taught me and provided me with guidance in the study of Economic as well as the data processing methods. Further, I would like to thank all my family members for their encouragement and accompany during the complement of this paper.

\section{REFERENCES}

[1] Mitford J. The American way of death[M]. New York: Simon and Schuster, 1978.

[2] Stahl D O. Oligopolistic pricing with sequential consumer search[J]. The American Economic Review, 1989: 700-712.

[3] Ellison G, Ellison S F. Search, obfuscation, and price elasticities on the internet[J]. Econometrica, 2009, 77(2): 427-452.

[4] Weian Li, Desheng Wu, Hao Xu. Reputation mechanism in online transactions: Evidence from Taobao $[\mathrm{J}]$. Nankai Management Review, 2007(05): 36-46. (in Chinese)

[5] Youzhi Xue, Yongfeng Guo. Research on the Competitive Strategy of C2C E-commerce Sellers: Based on the Analysis of Taobao.com [J]. Nankai Management Review, 2012, 15(05): 129-140. (in Chinese)

[6] Yali Dong, Bei Yang. Analysis of Influencing Factors of Consumer Buying Behavior under C2C E-commerce Platform [J]. Consumer Economics, 2007(03): 32-35+38. (in Chinese)
[7] Bertrand J. Théorie mathématique de la richesse sociale[J]. Journal des savants, 1883, 67(1883): 499-508.

[8] Diamond P A. A model of price adjustment[J]. Journal of economic theory, 1971, 3(2): 156168.

[9] Petrikaite V. Consumer obfuscation by a multiproduct firm[J]. The RAND Journal of Economics, 2018, 49(1): 206-223.

[10] Acemoglu D, Shimer R. Wage and technology dispersion[J]. The Review of Economic Studies, 2000, 67(4): 585-607.

[11] Janssen M C W, Moraga-González J L. Strategic pricing, consumer search and the number of firms $[\mathrm{J}]$. The Review of Economic Studies, 2004, 71(4): 1089-1118.

[12] Baye M R, Morgan J, Scholten P. Price dispersion in the small and in the large: Evidence from an internet price comparison site $[\mathrm{J}]$. The Journal of Industrial Economics, 2004, 52(4): 463-496.

[13] Zhou Wen-hui, Qiu Yun-jin, Jin Keke, Li Yuwen. The effect mechanism of e-commerce platform and bilateral market value cocreation on network effect: A case study based on Taobao [J]. Soft Science, 2015, 29(04): 8389. (in Chinese)

[14] Sun Puyang, Zhang Jingjia, Jiang Xiaoyu. Economic Research Journal, 2017, 52(07): 139-154. (in Chinese) 